\title{
Evaluation and Comparison of Battery Cell Balancing Methods
}

\author{
Bortecene Yildirim \\ School of Engineering \\ Newcastle University \\ Newcastle Upon Tyne, UK \\ Mohammed Elgendy \\ School of Engineering \\ Newcastle University \\ Newcastle Upon Tyne, UK
}

\author{
Andrew Smith \\ School of Engineering \\ Newcastle University \\ Newcastle Upon Tyne,UK
}

\author{
Volker Pickert \\ School of Engineering \\ Newcastle University \\ Newcastle Upon Tyne,UK
}

\begin{abstract}
Cell equalization is required in series connected strings to prolong battery lifespans, ensure safe operation, and to enhance the usable capacity of battery packs. Several cell balancing methods have been proposed in literature to address cell mismatch in series strings. Amongst these, dissipative balancing is the simplest and the most cost effective solution. However, this has a low efficiency and restricted balancing speed. Capacitor balancing is popular energy transferring method with its simple control, compact size, and low price; but it also has low balancing speed. Finally, runtime balancing, which is a relatively new technique, shows good performance in terms of balancing speed, and efficiency with its high cost and complex controller. Using MATLAB/ Simulink, this paper compares dissipative balancing, capacitive energy transferring balancing, and runtime balancing methods in terms of balancing speed, efficiency, complexity, and cost, with suitable applications mentioned.
\end{abstract}

Index Terms-Battery Storage, Battery Management Systems, Cell Equalization, Comparison of Cell Equalization

\section{INTRODUCTION}

Battery energy storage systems (BESS) are key components of smart grids, where their main purpose is to compensate for the stochastic nature of renewable power generation, and demand variations. As individual battery cell voltages vary from $1 \mathrm{~V}$ to $4.5 \mathrm{~V}$, cells are connected in series to increase the voltage levels of a battery pack. However, cell mismatch in series strings is inevitable due to manufacturing and thermal variations, differences in internal impedances, and self-discharging rates. As the performance of a battery pack is decided by the weakest cell in the string, the mismatch results in capacity reduction and wasted energy, during charging and discharging modes, respectively. In addition, battery lifetime is degraded, with a remote chance of explosion if the cells are not equalized [1]-[3].

To address cell mismatch, several balancing methods have been proposed in the literature. According to the way they process the redundant energy, the balancing methods can be divided into three main categories: dissipative balancing, energy transferring, and individual cell power control methods. In dissipative balancing techniques, balancing is achieved by dissipating the redundant energy of the higher charged cells in a resistor. This is the simplest and cheapest method, but it suffers from high balancing losses [4], [5]. Alternatively, energy shuttling techniques, which transfer energy from higher charged cells to lower charged cells, using storage elements, have higher balancing efficiencies than dissipative techniques. Amongst these, capacitor based energy shuttling methods are a more promising option because of their simpler controller, lower price, and compact size [6][9]. However, they have a low equalization speed. In contrast to dissipative and energy shuttling methods, runtime balancing methods are integrated into the power conversion stage. The cell balancing is achieved by controlling the power of the individual cells during charging and discharging, with the help of a power converter connected to each cell [10]-[12]. The main drawback of this method is its high cost.

All different balancing methods have their own advantages and disadvantages. In this paper the balancing methods are reviewed and compared in terms of balancing speed and efficiency, cost, control complexity and suitable application.

\section{BALANCING METHODS}

\section{A. Dissipative Balancing Method}

In the dissipative balancing method, equalization is achieved by dissipating excess energy from higher charged cells. Due to the parallel connected resistors across each cell (Fig. 1a), there will be continuous current flow through the resistors. Higher charged cells have higher voltages than lower charged ones, leading to more energy being dissipated from the higher charged cells, resulting in convergence of the voltages. However, there is still continuous power flows through the resistors even when all cells are balanced. To prevent continuous power losses, and to control the balancing circuits, a controllable switch has to be placed series with each resistor as shown in Fig. $1 b$.

Although this is the simplest and the cheapest equalization method, the excess energy is dissipated as heat, resulting in a low energy efficiency. In addition, there is no run time improvement with this method, and some of the available energy cannot be utilized in the string in discharging mode [7]-[9] 


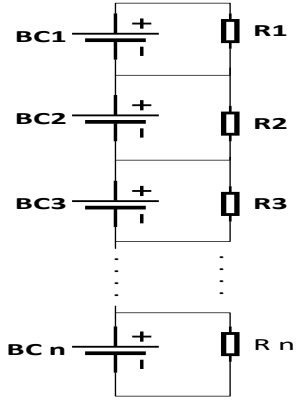

(a)

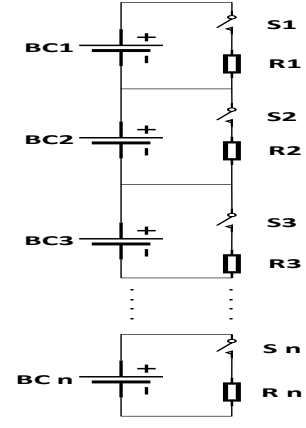

(b)
Figure 1. Dissipative Cell Balancing Circuits: a) Fixed Shunt Resistor, b) Switched Shunt Resistor

\section{B. Capacitor Based Energy Transferring Method}

Instead of dissipating the excess energy from higher charged cells, the energy is transferred to the lower charged cells via capacitors. The main advantages of this method over dissipating balancing methods are its higher efficiency, and utilization of the available energy. In the Switched Capacitor (SC) cell balancing method (Fig. 2a), capacitors connect between upper and lower cells in one switching cycle, enabling energy exchange between adjacent cells. All switches are controlled by complementary signals, and no voltage sensing and no closed loop control are required [6]-[8]. In this method, energy exchange is only possible for adjacent cells; therefore, the balancing takes a long time, with a low efficiency for long strings [8].

To improve the efficiency and reduce the balancing time, it is necessary to create paths for non-adjacent cells. This can be achieved by connecting extra capacitors between non-adjacent cells, such as the Double Tired SC [7], and Chain Structure Capacitor SC [8] methods. With these techniques, although the speed is increased, there is still no possibility for direct energy exchange between all cells. In [9], Ye, Cheng, Fong, Xue, and Lin proposed an optimized SC method to address this problem (Fig. 2d). With this method, direct energy transfer between all cells is possible, as all capacitors are connected with a common point; thus the balancing performance is independent of the number of series cells.

The control simplicity of capacitor based energy shuttling methods is promising. However, all capacitor based balancing methods rely on the voltage difference among cells, and their target to balance the voltage rather than the state of charge (SoC). This may result in increased unbalance due to the differences in the intrinsic resistances of the cells, and the equalization time, because of the flat discharge curve of the battery.

\section{Runtime Balancing Method}

In the modular battery structure (Fig. 3), each cell is connected to an individual low power converter, with each converter connected in series to increase the overall voltage. No central power converter is required to adjust the charging/discharging power. Cell balancing is achieved by adjusting the power of each module based on their individual SoC. Higher charged cells release more power than lower ones in the discharging mode, and lower charged cells are charged at a higher power in the charging mode. This system also enhances the system reliability, as faulty cells can be bypassed, with the rest of the modules supporting the DC bus [10], [11]. This control method is well summarized in [10]. However, compared to the other methods, the system is expensive due to the large number of power converters, and it requires a relatively complex controller.

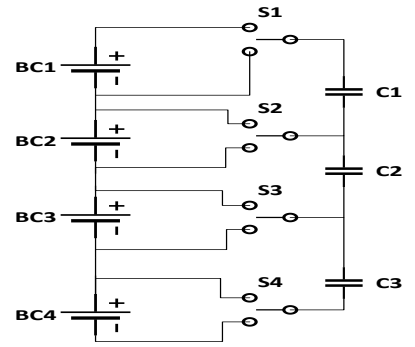

(a)

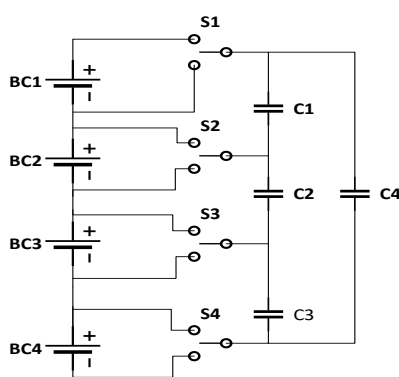

(c)

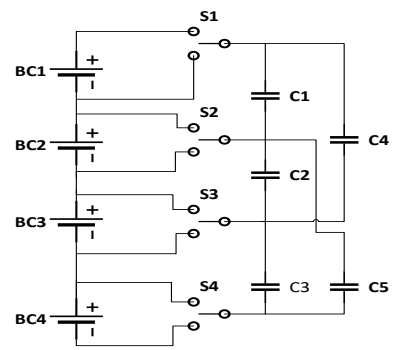

(b)

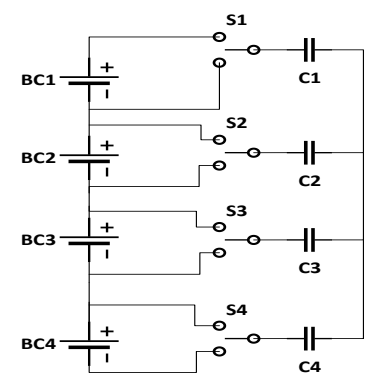

(d)
Figure 2. Capacitor Cell Balancing Circuits: a) Switched Capacitor (SC), b) Double Tiered SC, c) Chain Structure SC, d) Optimized SC

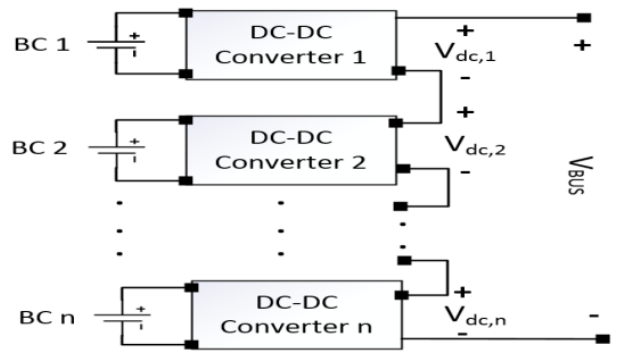

Figure 3. Runtime Cell Balancing Structure

\section{SIMULATION RESUlTS}

For the comparative simulations, 4 lithium batteries rated at $3.2 \mathrm{~V}$, and $10 \mathrm{Ah}$, were used. The cells initial conditions before balancing are shown in Table 1 .

Table I. CELL CONDITION AT THE BEGINNING OF SIMULATION
\begin{tabular}{|c|c|c|c|}
\hline Cell 1 & Cell 2 & Cell 3 & Cell 4 \\
\hline 70 & 65 & 68 & 60 \\
\hline
\end{tabular}

A. Switched Shunt Resistor Method (Fig 1.b)

There is no advantage in enabling balancing in discharging mode; therefore, the dissipative balancing is used for charging. For this simulation, the algorithm operates so that when one of the cells reaches it maximum SoC, the battery management 
system (BMS) stops the operation and balancing is enabled so all cell voltages are equal, then charging starts again.

The switched shunt resistor balancing with a $200 \mathrm{~mA}$ balancing current is illustrated in Figs. 4 and 5, and with a 1A balancing current in Figs. 6 and 7. With 200mA, balancing starts at 2450s and takes 5 hours; with $1 \mathrm{~A}$ it only takes 1 hour. However, the power loss of the individual cells during balancing is also 5 times higher with $1 \mathrm{~A}$ than $200 \mathrm{~mA}$ as the 5 times higher balancing current results in increased power loss. With the dissipative balancing the final $\mathrm{SoC}$ of the battery pack is equal to the weakest cell after balancing, which is 90 $\%$ in this case presented.

Dissipative balancing is the most common method due to its simplicity and low cost. However, in practice, the balancing current is restricted to several hundred $\mathrm{mA}$ to prevent excessive heat generation: and the balancing speed is too slow with this current value, especially for higher power applications. Therefore, it is a satisfactory balancing technique for low power applications and for a battery string composed of similar state of health $(\mathrm{SoH})$ cells.

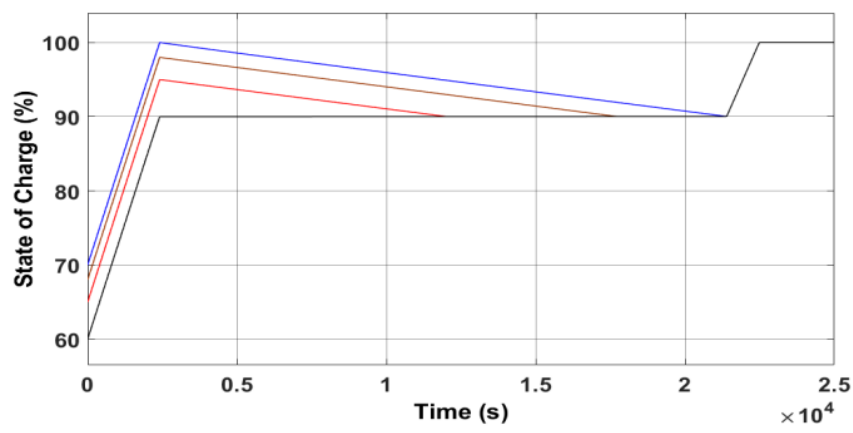

Figure 4. The cell SOCs with switched shunt resistor method, $200 \mathrm{~mA}$ balancing current
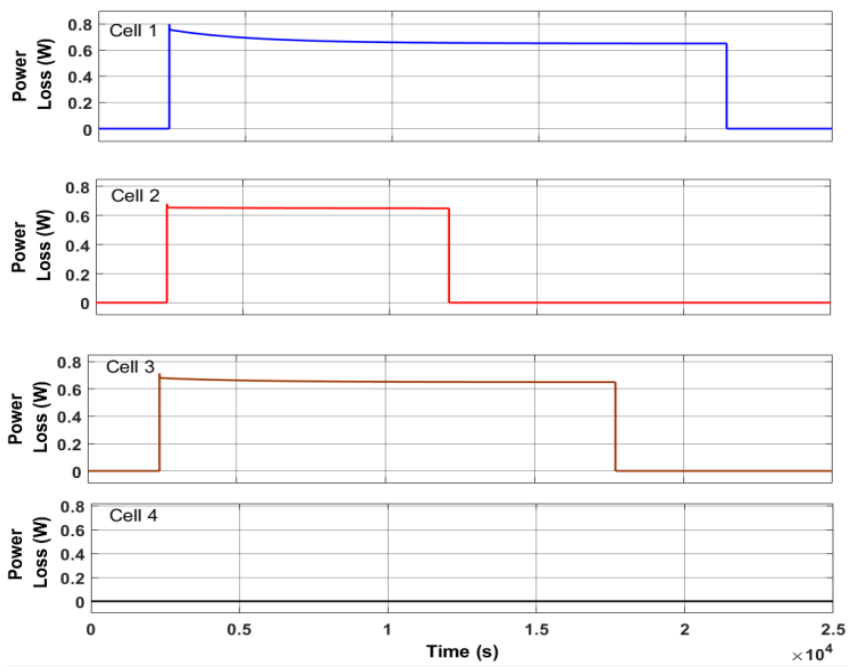

Figure 5. Power losses with switched shunt resistor method, $200 \mathrm{~mA}$ balancing current

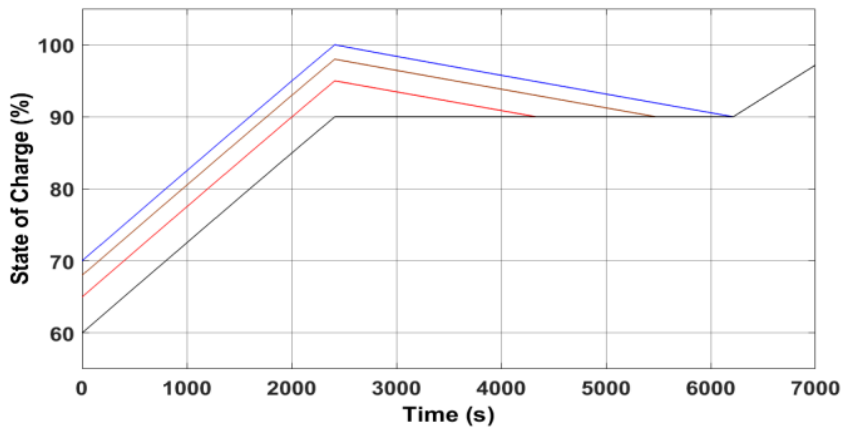

Figure 6. The cell SOCs with switched shunt resistor method, 1A balancing current
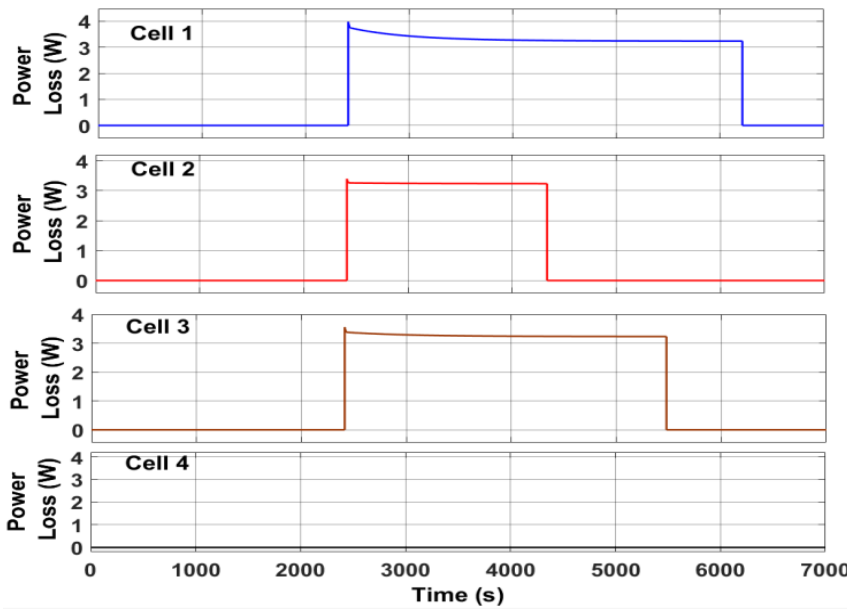

Figure 7. Power losses with switched shunt resistor method, 1A balancing current

\section{B. Switched Capacitor Method (Fig. 2)}

The simulations based on SC balancing methods have been performed with $470 \mu \mathrm{F}$ capacitors with an ESR of 20 $\mathrm{m} \Omega$ and $50 \mathrm{kHz}$ switching frequency. Figs. 8 and 9 show the simulation results with the SC and optimized SC balancing methods, respectively. It is clear that, unlike the standard SC method (Fig 2a), in which energy transfer is only possible between adjacent cells, direct energy transfer between all cells is possible with the optimized SC method (Fig $2 b$ ) and hence faster balancing can be achieved. Compared to the dissipative method, after balancing the average battery $\mathrm{SoC}$ is equal to the average $\mathrm{SoC}$ of battery pack with energy transferring method if power losses are ignored. The SoC of each cell after balancing is equal to average $\mathrm{SoC}$ of series string which is 65.75 before balancing.

For high power applications the active balancing is a suitable method to prolong battery service time with its relatively low price compared to the runtime balancing method.

\section{Individual Cell Power ControlMethod}

A bidirectional half bridge DC-DC buck-boost converter, with $250 \mathrm{kHz}$ switching frequency, $6.8 \mu \mathrm{H}$ inductor and $33 \mu \mathrm{F}$ capacitor is used in the simulation analysis. With this method, in order to achieve balancing, the lower charged cells are discharged with a lower power in discharging mode. Since cell 4 has the lowest energy, it has the lowest dissipation during balancing, while, cell 1 has the highest one since it is the 
highest charged cell. As the DC side current is same for all modules at the series connected side the voltage difference among the modules creates a power difference (Figs. 10 and 12). The sum of the module voltages is always equal to the DC bus voltage which, in this case, is $24 \mathrm{~V}$. In this method the equalization speed is directly related to the battery load, with higher battery load giving faster balancing as demonstrated in Figs. 11 and 13.

The runtime balancing is an appropriate balancing technique for a system based on pre-used batteries, where high imbalance is expected as the continuous balancing during battery operation prevents the divergence of the cell voltage, even if the pack composed of different SoH cells.

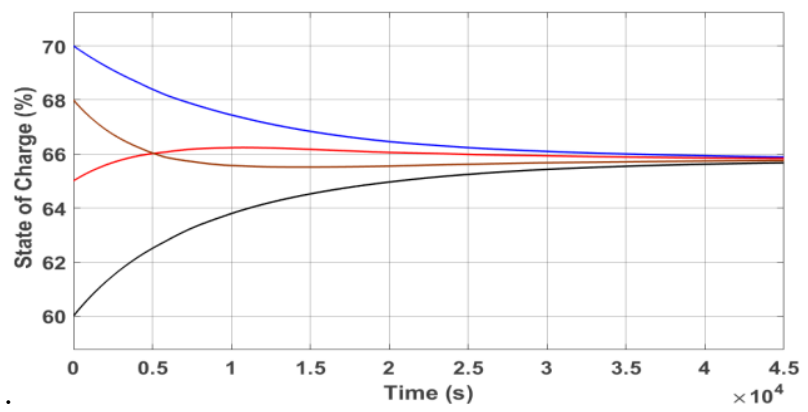

Figure 8. The cell SoCs with SC

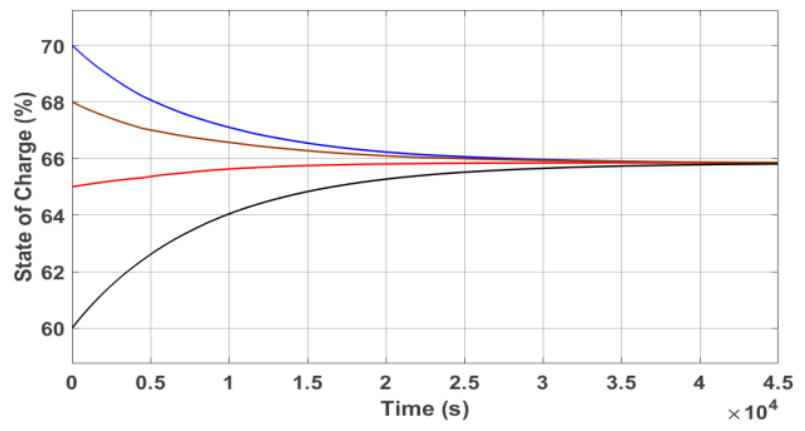

Figure 9. The cell SoCs with optimized SC

\section{ANALYSES COMPARISONS}

Each of the balancing methods analyzed are different from each other in terms of their equalization method, balancing speed, efficiency, and control complexity and these will be discussed below.

\section{A. Balancing Speed}

In dissipative balancing methods, the balancing speed is directly related to the balancing current. For a faster balancing speed higher currents in the resistors are required which increase the power loss, and means more advanced cooling systems are required. In optimized SC method, unlike the SC method, the balancing speed is independent of the number of cells. However, as all capacitor based energy shuttling methods rely on the voltage difference between cells; they both show poor performance in terms of equalization speed. With dissipative and energy shuttling balancing methods the balancing speed is independent of battery load. However, in runtime balancing method the equalization speed is quick at high loads as the DC bus current is high, providing higher power differences amongst the modules with the same voltage differences.
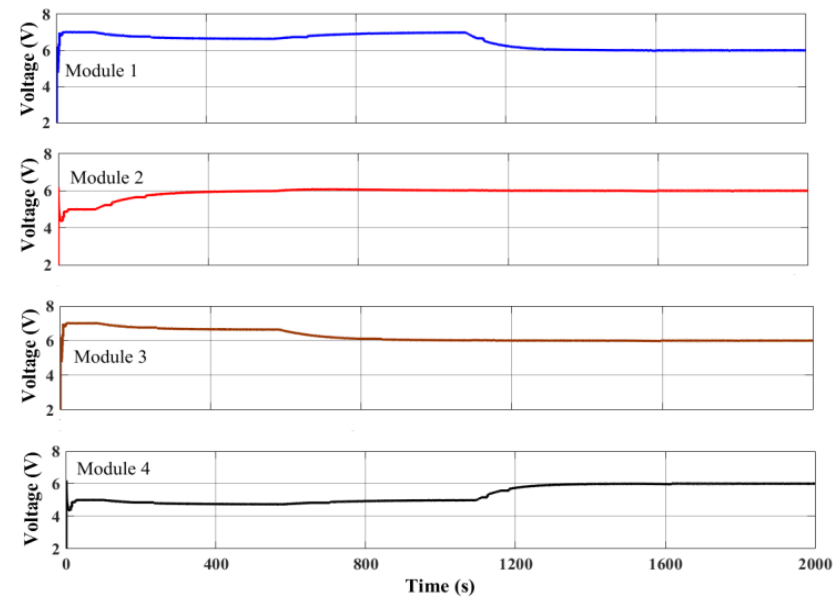

Figure 10. The voltage of each module at the series connected side with $0.8 \mathrm{C}$ battery load

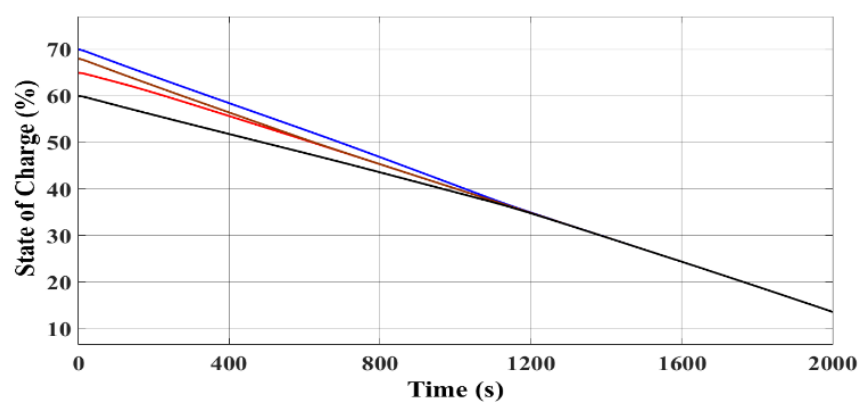

Figure 11. Convergence of cells SoC with $0.8 \mathrm{C}$ battery load
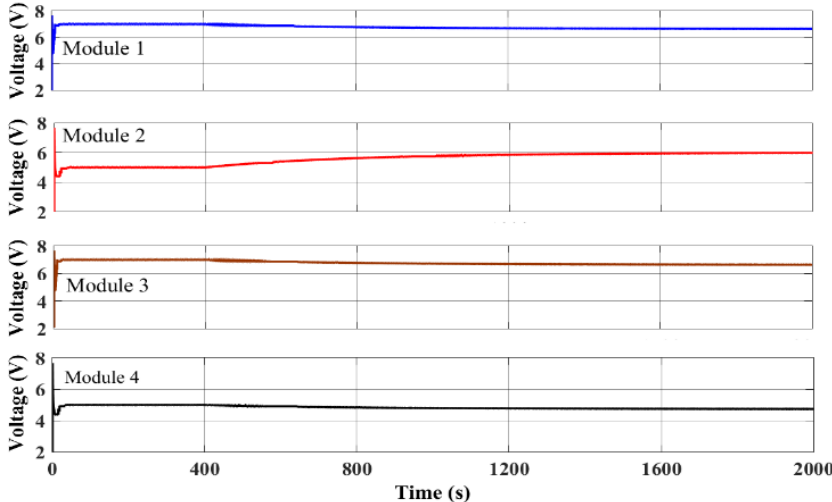

Figure 12. The voltage of each module at the series connected side with $0.2 \mathrm{C}$ battery current

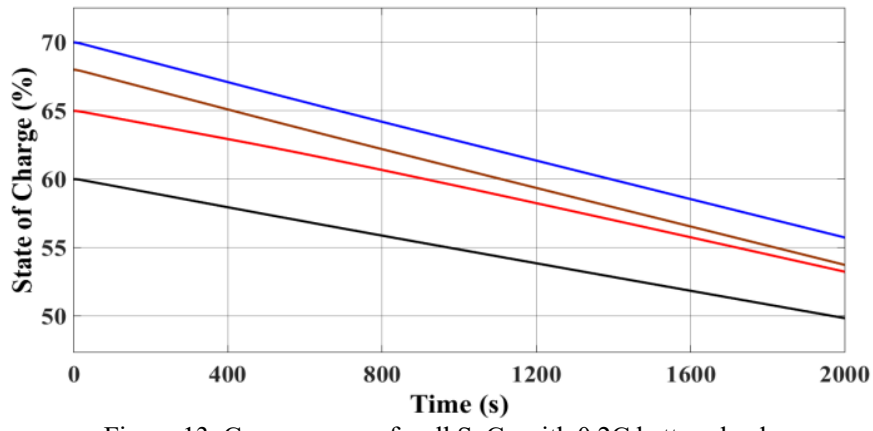

Figure 13 . Convergence of cell SoCs with $0.2 \mathrm{C}$ battery load 
This power difference is shown in (1). Among all balancing types, the runtime method achieves the fastest equalization.

$$
\Delta P=I_{d c} * \Delta V_{d c}
$$

where $\triangle P$ is the power difference among cells, $I_{d c}$ is the $d c$ bus current. and $\Delta V_{d c}$ is the converter voltage differences at the series connected side.

\section{B. Balancing and System Efficiency}

In the dissipative balancing method, the excess energy from the higher charged cells is wasted, thus the energy efficiency of dissipative balancing is zero [7]. On the other hand, the energy efficiency of the SC method is around $97 \%$ with three cells [7]; but, when the string cell number increases, the efficiency decreases.

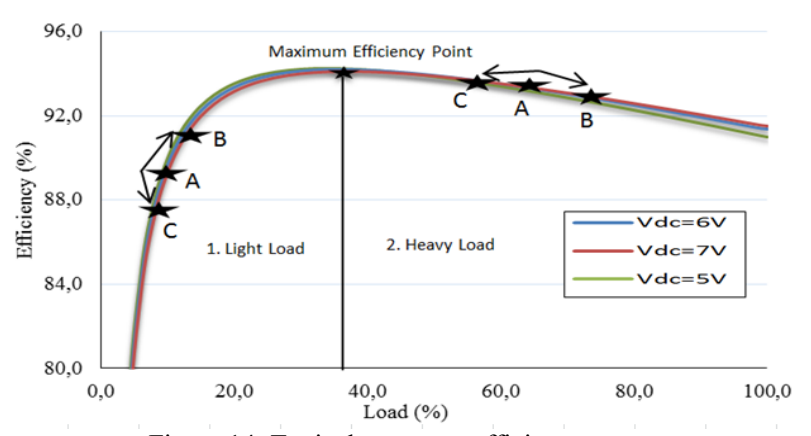

Figure 14. Typical converter efficiency curve

BESS with dissipative and energy shuttling balancing techniques have two independent losses; conversion, and balancing and these can be evaluated separately. In contrast to the dissipative and energy shuttling methods, the efficiency of the runtime balancing method cannot be evaluated alone, as the cell balancing and power processing features are integrated. However, by analyzing the effect of balancing on conversion efficiency it is possible to estimate the balancing efficiency. Fig. 14 shows the typical efficiency curve of a DC-DC converter, and in order to evaluate the effect of balancing the curve is divided into two areas from its maximum efficiency point. When all cells are equally charged, they share the power evenly and all converters operate at point A. If there is a mismatch among the cells, some of the modules operate at point $\mathrm{B}$, and some of them operate at point $\mathrm{C}$, and the new system efficiency can be calculated using (2).

$$
\eta_{\text {system }}=\sum_{\mathrm{k}=1}^{4} \eta_{\mathrm{k}}{ }^{*} \mathrm{w}_{\mathrm{k}}
$$

where $\eta_{\text {system }}$ and $\eta_{k}$ are the system efficiency and individual modules efficiency, respectively, and $w_{k}$ is the weighting factor of each module.

With heavy load (right hand side) the power is processed less efficiently, as the efficiency of point B is lower than that of point $\mathrm{A}$, indicating a reduction in system conversion efficiency due to the balancing. With light loads (left hand side), the power is processed more efficiently as the point $\mathrm{B}$ has a higher efficiency than point A. It shows that balancing has positive impact on the conversion efficiency.

\section{CONCLUSION}

In this paper, cell balancing methods have been compared using MATLAB/Simulink. Dissipative balancing is the simplest and most cost effective method, but its efficiency is low and it is not suitable for higher power applications. For capacitive balancing, the voltage based balancing feature is its main drawbacks, limiting its balancing speed and accuracy. Finally, runtime balancing has fast and efficient balancing features, and is also appropriate for pre-used batteries, but with added complexity and increased cost. In summary, each balancing method has its own advantages and disadvantages, with their choice dependent on the systems cost and application.

\section{REFERENCES}

[6] M. Evzelman, M. M. U. Rehman, K. Hathaway, R. Zane, D. Costinett, and D. Maksimovic, "Active Balancing System for Electric Vehicles With Incorporated Low-Voltage Bus," IEEE Transactions on Power Electronics, vol. 31, pp. 7887-7895, 2016.

[7] A. C. Baughman and M. Ferdowsi, "Double-Tiered SwitchedCapacitor Battery Charge Equalization Technique," IEEE Transactions on Industrial Electronics, vol. 55, pp. 2277-2285, 2008.

[8] M. Kim, C. Kim, J. Kim, and G. Moon, "A Chain Structure of Switched Capacitor for Improved Cell Balancing Speed of Lithium-Ion Batteries," IEEE Transactions on Industrial Electronics, vol. 61, pp. 3989-3999, 2014.

[9] Y. Ye, K. W. E. Cheng, Y. C. Fong, X. Xue, and J. Lin, "Topology, Modeling, and Design of Switched-Capacitor-Based Cell Balancing Systems and Their Balancing Exploration," IEEE Transactions on Power Electronics, vol. 32, pp. 4444-4454, 2017.

[10] W. Huang and J. A. A. Qahouq, "Energy Sharing Control Scheme for State-of-Charge Balancing of Distributed Battery Energy Storage System," IEEE Transactions on Industrial Electronics, vol. 62, pp. 2764-2776, 2015.

[11] Y. Li and Y. Han, "A module-integrated distributed battery energy storage and management system," IEEE Transactions on Power Electronics, vol. 31, pp. 8260-8270, 2016. M. Evzelman, M. M. U. Rehman, K. Hathaway, R. Zane, D. Costinett, and D. Maksimovic, "Active Balancing System for Electric Vehicles With Incorporated Low-Voltage Bus," IEEE Transactions on Power Electronics, vol. 31, pp. 7887-7895, 2016. 